Article

\title{
The Potential of a Hair Follicle Mesenchymal Stem Cell-Conditioned Medium for Wound Healing and Hair Follicle Regeneration
}

\author{
Keng-Liang Ou 1,2,3,4,5, Yun-Wen Kuo ${ }^{6,+}$, Chia-Yu Wu ${ }^{7,8}$, Bai-Hung Huang 4,9,10, \\ Fang-Tzu Pai ${ }^{4,8}$, Hsin-Hua Chou ${ }^{11,12}$, Takashi Saito ${ }^{13}$ (D), Takaaki Ueno ${ }^{3}$, Yung-Chieh Cho ${ }^{4,11}$ \\ and Mao-Suan Huang ${ }^{1,14, *}$ \\ 1 Department of Dentistry, Taipei Medical University-Shuang Ho Hospital, New Taipei City 235, Taiwan; \\ klouyu@gmail.com \\ 2 Department of Oral Hygiene Care, Ching Kuo Institute of Management and Health, Keelung 203, Taiwan \\ 3 Department of Dentistry and Oral Surgery, Osaka Medical College, Osaka 569-8686, Japan; \\ ueno@osaka-med.ac.jp \\ 4 Biomedical Technology R \& D Center, China Medical University Hospital, Taichung 404, Taiwan; \\ babyfireh@gmail.com (B.-H.H.); M249108004@tmu.edu.tw (F.-T.P.); D204106003@tmu.edu.tw (Y.-C.C.) \\ 5 3D Global Biotech Inc. (Spin-off Company from Taipei Medical University), New Taipei City 221, Taiwan \\ 6 Division of Prosthodontics, Department of Dentistry, Taipei Medical University Hospital, Taipei 110, Taiwan; \\ kjpopo@hotmail.com \\ 7 Division of Oral and Maxillofacial Surgery, Department of Dentistry, Taipei Medical University Hospital, \\ Taipei 110, Taiwan; borgiawu@gmail.com \\ 8 School of Dental Technology, College of Oral Medicine, Taipei Medical University, Taipei 110, Taiwan \\ 9 Asia Pacific Laser Institute, New Taipei City 220, Taiwan \\ 10 Implant Academy of Minimally Invasive Dentistry, Taipei 106, Taiwan \\ 11 School of Dentistry, College of Oral Medicine, Taipei Medical University, Taipei 110, Taiwan; \\ hhchou@tmu.edu.tw \\ 12 Dental Department of Wan-Fang Hospital, Taipei Medical University, Taipei 116, Taiwan \\ 13 Division of Clinical Cariology and Endodontology, Department of Oral Rehabilitation, School of Dentistry, \\ Health Sciences University of Hokkaido, Hokkaido 061-0293, Japan; t-saito@hoku-iryo-u.ac.jp \\ 14 School of Oral Hygiene, College of Oral Medicine, Taipei Medical University, Taipei 110, Taiwan \\ * Correspondence: hms4837@tmu.edu.tw \\ + Co-first author: Yun-Wen Kuo.
}

Received: 11 March 2020; Accepted: 9 April 2020; Published: 11 April 2020

Abstract: The study elucidated the wound healing and hair regeneration properties of a conditioned medium prepared from the culture of human hair follicle mesenchymal stem cells (HFMSCs). The wound-healing effects of mesenchymal stem cell-conditioned medium (MSC-CM) were tested in vitro using scratch assays co-cultured with $\mathrm{HaCaT}$ keratinocyte and monitored through optical microscopy. The cell proliferation of HFMSCs and the HaCaT keratinocyte were observed in the presence of different kinds of drugs including UK5099, sodium L-lactate, lactate dehydrogenase-A, MSC-CM, caffeine, and caffeic acid. The hair regeneration properties were investigated in vivo by administrating the MSC-CM solutions to adult B6 mouse models. For quantification, hematoxylin and eosin staining were performed following euthanasia. In vitro results revealed that MSC-CM promotes dermal cell migrations and enhances proliferation of HFMSCs and HaCaT keratinocytes, demonstrating wound-healing properties. Moreover, when the MSC-CM solutions were applied to the shaved mouse skin, a dark area that expanded overtime was seen. Although no hair growth was found, histological analysis proved that a fat layer thickness increment was found under the mouse's skin, ultimately projecting the formation of new hair growth. MSC-CM promotes the migration and proliferation of dermal keratinocytes that are beneficial for wound healing and hair growth. It is believed that MSC-CM can potentially serve as the basis of alternative therapeutic applications for 
wound closure and skin regeneration as well as hair growth stimulation and hair loss prevention in alopecia.

Keywords: hair follicle; hair regeneration; mesenchymal stem cells; wound healing

\section{Introduction}

Skin injuries are the most commonly found injury caused by blisters, burns, cuts, and ulcers of various severities [1]. If left untreated, any skin injury can interfere with its protective function and cause body tissues to be easily exposed to mechanical damages and microbial infections [2]. Other problems that may accompany wound healing are excessive healing, delayed wound healing, or lack of skin appendages [1]. Recently, the increasing demand for quality improvement of damaged skin has led to the shifting focus of wound repair to the restoration of anatomy and function of the skin [3]. Thus, identifying an effective approach to enhance the wound healing process is indispensable [1-4].

Traditionally, the main purpose of wound care is to obtain wound closure at the earliest time [1]. Nowadays, the use of stem cell-based regenerative therapy to renew physiological tissue and promote the regeneration of various types of wounds is increasingly popular [2,5]. Mesenchymal stem cells (MSCs) have been reported to have therapeutic potential after skin injury and extensive proliferation and differentiation capacity that have the ability to suppress the stimulation of immune cells and participate in tissue repair and regeneration [5-8]. MSC releases a combination of growth factors and cytokines, so-called secretome, which can be applied to the wound through a conditioned medium (CM) $[5,9,10]$. The cytokines and growth factors play a crucial role in activating and stimulating fibroblast proliferation during the wound healing process [11]. Previous studies have reported positive results of wound healing and skin repair by utilizing CM from cultured MSCs (MSC-CM) derived from different sources such as bone marrow mesenchymal stem cell (BM-MSC) [9,12,13], adipose stem cell [14-16], Wharton's jelly-derived MSC [17], and human umbilical MSC [1]. Furthermore, numerous studies have proven that several kinds of drugs can be potentially used to promote cell growth and proliferation that is beneficial for wound healing and hair regeneration including UK5099 [18], sodium L-lactate (SLL) [19], lactate dehydrogenase-A (LDHA) [18], caffeine [20,21], and caffeic acid (CFA) [22].

Related to MSC-CM, not only can it be used in the repair of skin defects or wounds, but it can also enhance hair regrowth through differentiation or release of growth factors [23,24]. A study by Yoon et al. [25] revealed surprising hair growth at the wound site following wound healing after MSC-CM was injected subcutaneously around a full-thickness wound in mice. To date, available data regarding the use of MSC-CM for hair follicle regeneration as well as wound healing are still limited, especially when using human hair follicle mesenchymal stem cells (HFMSCs) as the main source. Even though HFMSCs have been shown to have potential in hair regrowth, further studies are required to identify culture conditions that trigger the hair stimulating factors that warrant this application. Thus, to elucidate the regenerative potential of MSC-CM, we investigated CM from the supernatant of cultured HFMSCs and evaluated their effects on wound closure and hair regeneration.

\section{Materials and Methods}

\subsection{Isolation of HFMSCS}

To acquire HFMSCs, donor hair follicles were uniformly obtained from the adult occipital scalp by the ARTAS $^{\circledR}$ robotic system (San Jose, CA, USA). Isolation techniques were designed according to the previously established protocol with some modifications. First, the biopsy sample of hair follicles was thoroughly rinsed with phosphate-buffered saline (PBS; Sigma, Taipei, Taiwan) containing penicillin/streptomycin $(\mathrm{P} / \mathrm{S})$, then the excess adipose tissues were removed. After that, the samples were treated with trypsin/ethylenediaminetetraacetic acid (EDTA; Sigma, Taipei, Taiwan) at $37^{\circ} \mathrm{C}$ 
for $30 \mathrm{~min}$, and then digested with dispase at $4{ }^{\circ} \mathrm{C}$ for over $16 \mathrm{~h}$. After the digestion procedure, the hair and scalp portions were separated from the hair follicles using fine forceps, then filtered through a $70 \mu \mathrm{m}$ cell strainer, and washed extensively with PBS. Prior to the proliferation assay, the cells were cultured in stromal medium (Dulbecco's Modified Eagle Medium and Ham's F-12 medium (DMEM-F12; Gibco, Taipei, Taiwan)) and 10\% fetal bovine serum (FBS; Gibco, Taipei, Taiwan) for seven days. After seven days, cells from individual wells were harvested by $0.25 \%$ trypsin in $0.01 \%$ EDTA following centrifugation at $800 \mathrm{rpm}$ for $5 \mathrm{~min}$. The centrifuged liquid was filtered through a $100 \mu \mathrm{m}$ cell strainer. Then, the hair and scalp were separately transferred into a sterilized 6-well plate and maintained in MSCs culture medium (100 $\mu \mathrm{L}$ DMEM, 10\% FBS, 1\% P/S, $2.45 \mathrm{~g} / \mathrm{L}$ sodium bicarbonate, and $10 \mathrm{ng} / \mathrm{mL}$ human epidermal growth factor). The cells were maintained in a culture dish for two weeks and the medium was replaced every three days.

\subsection{Cell Culture}

The HaCaT keratinocyte cell line was obtained from the National Health Research Institutes, Miaoli, Taiwan. The cells were maintained in DMEM-High Glucose (DMEM-HG; Gibco, Taipei, Taiwan) culture medium, supplemented with $10 \%$ FBS and $1 \%$ antibiotics (P/S) at $37{ }^{\circ} \mathrm{C}$ and in a humidified atmosphere containing $5 \% \mathrm{CO}_{2}$. The MSC-CM was generated from $80 \%$ confluent HFMSCs in $100 \mathrm{~mm}$-diameter culture dishes fed with $5 \mathrm{~mL}$ of DMEM-F12 and incubated at $37{ }^{\circ} \mathrm{C}$ in an atmosphere of $5 \% \mathrm{CO}_{2} / 95 \%$ air for $72 \mathrm{~h}$. At the end of the incubation period, the media were collected and centrifuged at $300 \times g$ for $10 \mathrm{~min}$ to avoid contamination of cell fragments and the supernatant. After the centrifugation, the supernatant that was used as MSC-CM was harvested and added to the $\mathrm{HaCaT}$ keratinocyte. The supernatant incubated with $80 \%$ confluent HFMSC for $72 \mathrm{~h}$ and processed in the above-mentioned steps was quantified as $1 \mathrm{U}$ concentration of MSC-CM.

\subsection{Scratch Wound Assay}

Confluent monolayer HaCaT keratinocyte cells in six-well culture plates were fed with DMEM-HG and maintained for $24 \mathrm{~h}$ at $37^{\circ} \mathrm{C}$ and $5 \% \mathrm{CO}_{2}$. After $24 \mathrm{~h}$, the monolayer was scratched with a sterile plastic micropipette tip ( $200 \mu \mathrm{L}$ pipette tip) approximately $0.4-0.5 \mathrm{~mm}$ in width. The cells were washed three times with PBS, then DMEM-F12 (control medium) or MSC-CM was added. Photographs of the wounds were taken at $0 \mathrm{~h}, 24 \mathrm{~h}$, and $48 \mathrm{~h}$ by FV1000/IX81 fluorescence microscope (Olympus, Tokyo, Japan) and the width of the healed area was measured by the ImageJ analysis program (National Institutes of Health, Bethesda, MD, USA). All scratch assays were performed in four independent experiments.

\subsection{Proliferation Analysis}

HFMSC and HaCaT keratinocytes were seeded in 12-well culture plates, then the starvation process was performed for $24 \mathrm{~h}$ in which the cells were fed with serum-free medium without FBS. After that, various kinds and concentrations of drugs such as UK5099: $5 \mu \mathrm{M}, 10 \mu \mathrm{M}, 20 \mu \mathrm{M}$; SLL: $5 \mathrm{mM}, 10 \mathrm{mM}, 20 \mathrm{mM}$; LDHA: $100 \mathrm{U} / \mathrm{mL}, 1 \mathrm{KU} / \mathrm{mL}, 10 \mathrm{KU} / \mathrm{mL}$; MSC-CM: $0.01 \mathrm{U}, 0.05 \mathrm{U}, 0.1 \mathrm{U}$; caffeine: $0.001 \%, 0.01 \%, 0.10 \%$; CFA: $10 \mathrm{Um}, 50 \mu \mathrm{M}, 100 \mu \mathrm{M}$ were added and the cells were incubated for $24 \mathrm{~h}$. The viability of HFMSC and HaCaT keratinocyte cells treated with different drugs was evaluated and captured using an FV1000/IX81 fluorescence microscope. For immunofluorescence staining, the cells were incubated with diluted primary antibodies against the anti-Ki67 antibody (Abcam ab15580, Cambridge, MA, USA), according to the manufacturer's instructions. The primary antibody was detected by using a biotinylated goat anti-rabbit antibody (Abcam ab150077, Cambridge, MA, USA). The quantitative data were measured by the ImageJ analysis software (National Institutes of Health, Bethesda, MD, USA) from four independent experiments. 


\subsection{HFMSC Culture Solution Administered to Animal Skin}

This study was conducted using a total of four adult B6 mice as the animal models (National Laboratory Animal Center No. 107-NLAC-EN-007, New Taipei City, Taiwan). To obtain precise data, the vehicle and MSC-CM were treated on the same mice on different sides of the body. Specifically, before the experiment, the left and right body of each rat was shaved as close as possible to the skin and were randomly divided into two groups (treated group and control group) according to the gel to be applied. In all mice, the left side of the body was smeared with the first gel (vehicle or MSC-CM), while the right side of the body was smeared with the second gel (vehicle or MSC-CM). The growth of the hairs was evaluated on the skin of the mice and pictures were taken every five days. After 15 days of observations, the animal was sacrificed for quantitative analysis. Each of the experiments was performed in quadruplicate.

\subsection{Histology Staining}

Following euthanasia, the skin was directly fixed in 10\% formalin at room temperature for $24 \mathrm{~h}$. The tissues were then embedded in paraffin, sectioned, and mounted on glass microscope slides. The sections were stained with hematoxylin and eosin (HE), then examined via an Aperio CS digital image capture pathology scanner (Leica Biosystems, Buffalo Grove, IL, USA) under different magnifications.

\subsection{Statistical Analysis}

Data were analyzed using the paired t-test and presented as means \pm standard deviation. The differences were considered as statistically significant with the $p$-value $<0.05$.

\section{Results}

\subsection{MSC-CM Promotes Wound Healing}

Figure 1 displays the progression of wound closure in the scratch assay of the HaCaT keratinocyte in the presence of MSC-CM from the time of scratching until $72 \mathrm{~h}$ after the scratch was performed under a phase-contrast microscope. Clearly, the width of the healed area of the HaCaT keratinocyte treated sample was more rapidly closed than that of the untreated sample in $48 \mathrm{~h}$ and $72 \mathrm{~h}$ of observation. A similar result was also seen from the quantitative evaluation (Figure 2) when the HaCaT keratinocyte scratch assays were performed in MSC-CM, where there was a significantly enhanced rate of cell migration compared to the control. To be more specific, when the cell migration of the HaCaT keratinocyte scratch assays was $45 \%$ in the control medium (at the $48 \mathrm{~h}$ time point), it was almost $60 \%$ in the presence of MSC-CM. At the $72 \mathrm{~h}$ time point of the experiments, when the cell migration rate of the $\mathrm{HaCaT}$ keratinocyte scratch assays was $70 \%$ in the presence of MSC-CM, it was only $50 \%$ in the control medium. These findings indicate that MSC-CM enhanced the rate of wound closure of the $\mathrm{HaCaT}$ keratinocyte by promoting cell migration during wound healing.

To examine whether MSC-CM could stimulate cell survival and proliferation during wound closure, the HaCaT keratinocyte and HFMSC were treated with different kinds and concentrations of drugs. Given the above-mentioned reasons, these drugs have the ability to improve cell growth and proliferation, thus, in this study, we used them as positive controls compared to our MSC-CM. After the treatment, the Ki67 (proliferation marker) positive cells were found more in the HFMSC and HaCaT keratinocyte treated groups compared to control (Figure 3). The quantitative study from Figure 4 shows that among all kinds of drug treatments, in both the HFMSC and HaCaT keratinocyte, the group treated with MSC-CM showed the noticeably highest cell proliferation compared to other drugs. The results indicate that the conditioned medium of MSC at low to high concentrations can effectively promote the proliferation of the HFMSC and HaCaT keratinocyte, supporting the progression of wound healing. 


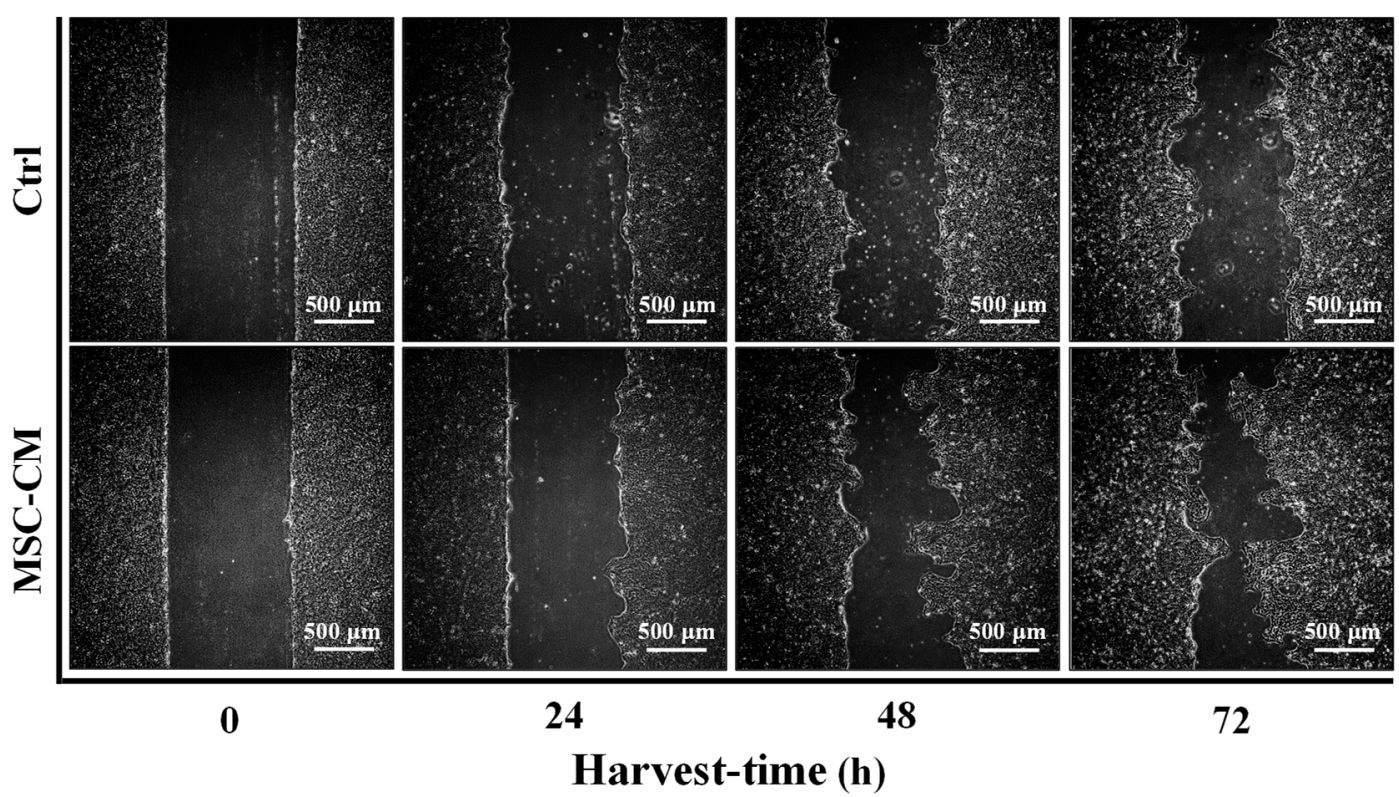

Figure 1. Optical micrographs showing the progression of wound closure in scratch assays of HaCaT in the presence of MSC-CM at different observational periods.

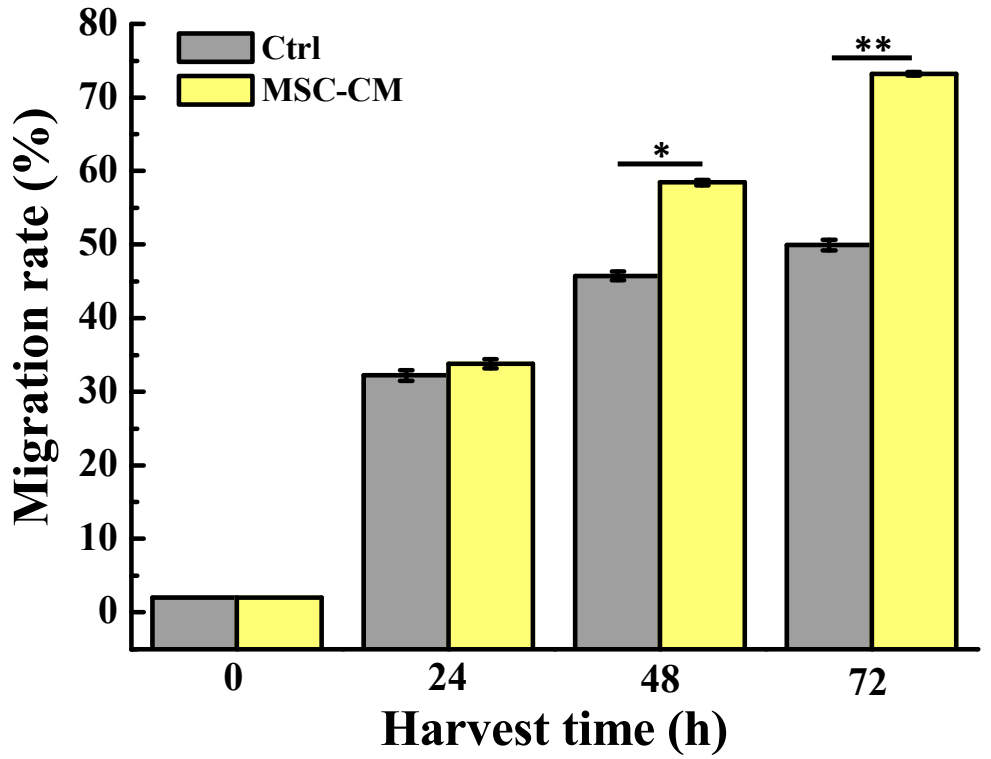

Figure 2. Quantitative evaluation of the wound healing reflecting the migration rate of the cells around the wound at various time intervals $\left({ }^{*} p<0.05\right.$ and $\left.{ }^{* *} p<0.01\right)$. 

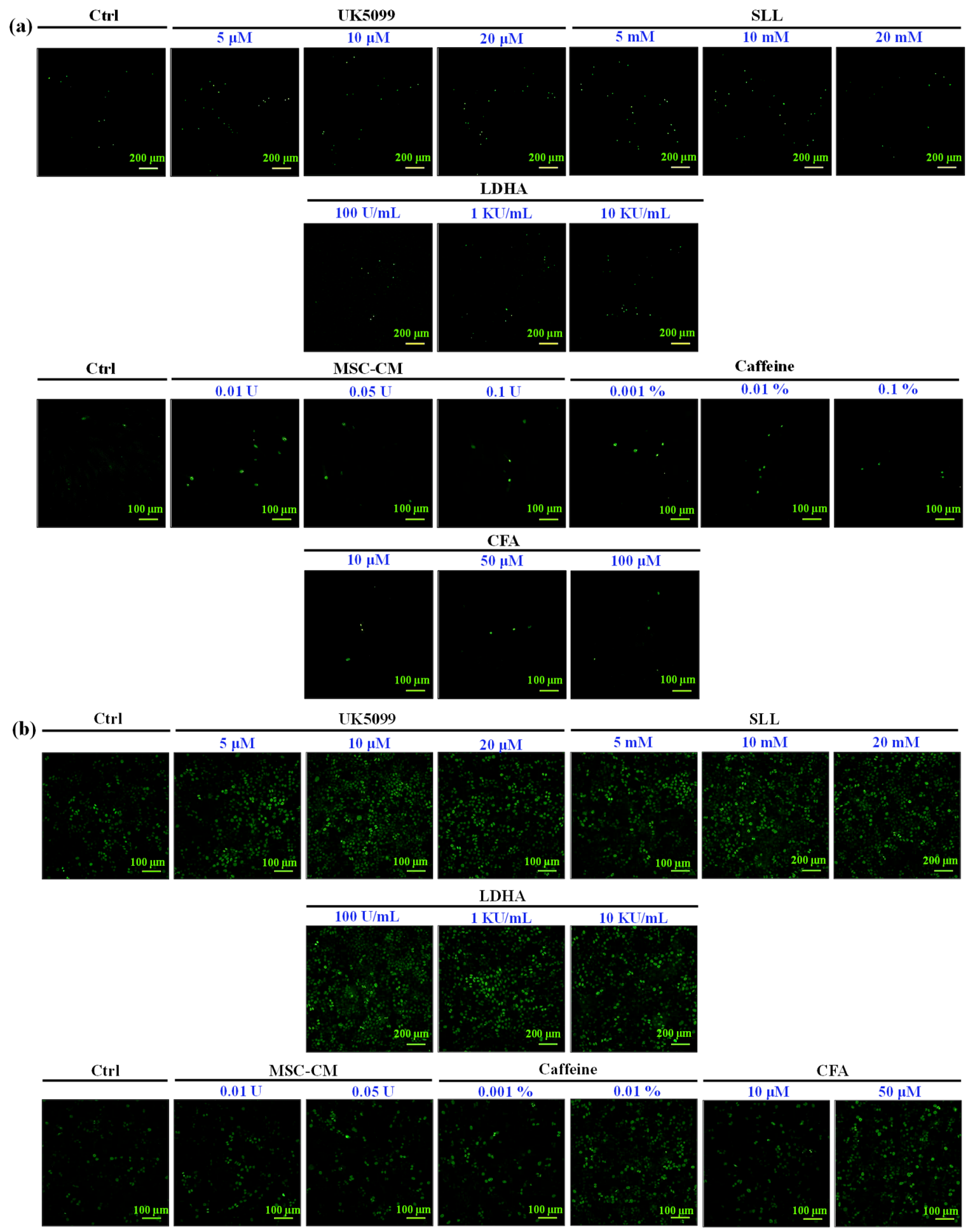

Figure 3. Ki67 staining of (a) HFMSC and (b) HaCaT after being treated with different kinds of drugs. More Ki67 positive cells were observed in both the HFMSCs and HaCaT treated samples. 

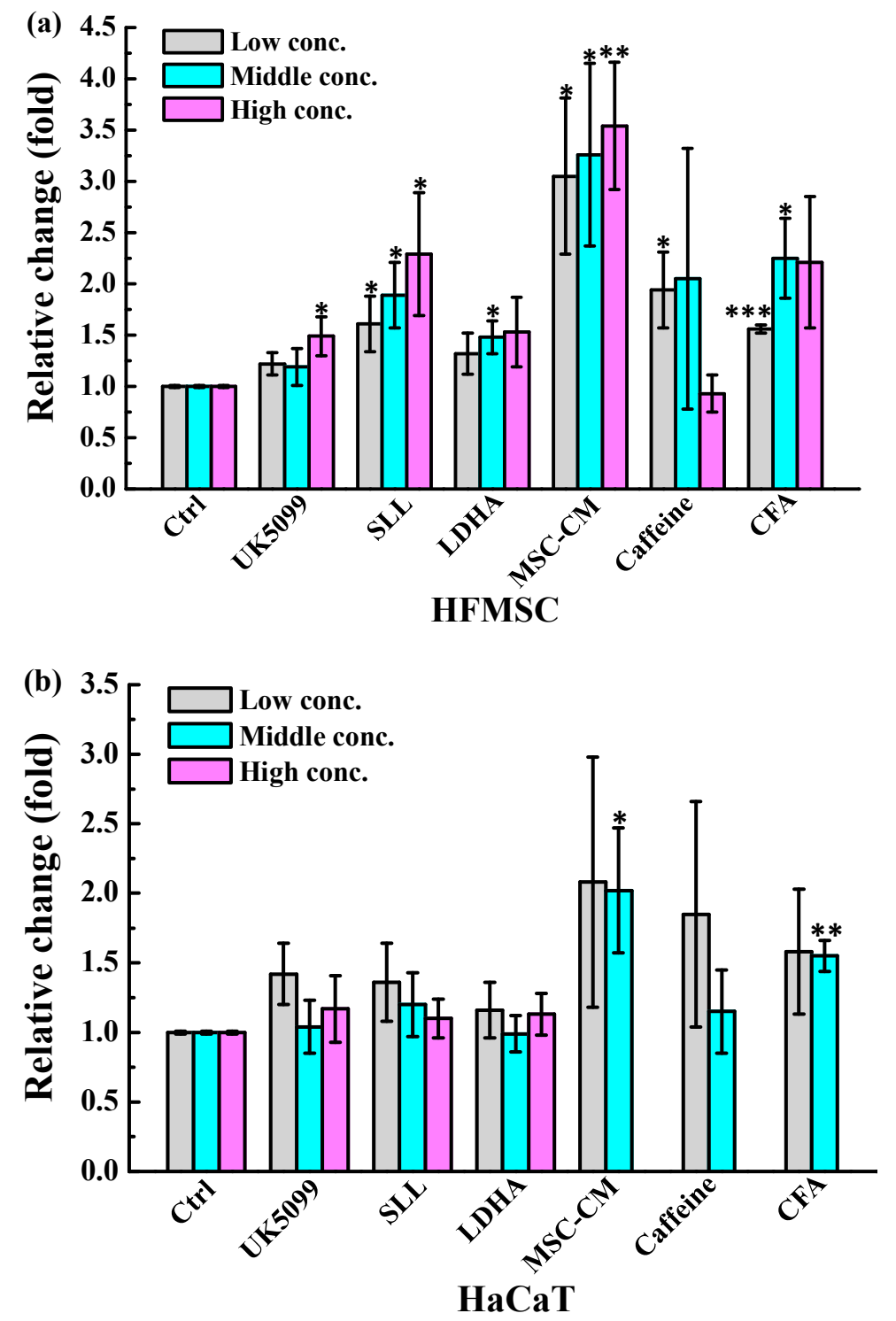

Figure 4. The statistical analysis of Ki-67 expression in (a) HFMSC and (b) HaCaT treated with UK5099, SLL, LDHA, MSC-CM, caffeine, and CFA in certain concentrations $\left({ }^{*} p<0.05,{ }^{* *} p<0.01\right.$ and $* * * p<0.001)$.

\subsection{MSC-CM Potential for Hair Regeneration}

To determine whether conditioned medium from HFMSC affected the hair follicle cycle and growth, we collected the culture medium from HFMSC and administrated it to the skin of mice with DMEM-F12 used as the control. Figure 5 depicts a photographic image taken from each sample from day 0 to day 15 after the MSC-CM treatments. After the left and right body of the mice were respectively subjected with the vehicle and MSC-CM, a blue to black area began to appear on the skin surface in which the area expanded or the color became darker with time. Even though no significant hair growth was observed at the end of the test, apparently, the MSC-CM treatment on day 15 highlighted a darker area of the skin and bigger diffusion. To confirm these findings, we harvested the skin of the mice for histological analysis with HE staining. Figure 6 denotes the hair follicle cycling was enlarged in the MSC-CM treated group when compared to the vehicle. The results represent that MSC-CM may promote hair growth by enhancing hair follicle cycling in the left and right body skin of mice. Figure 7 shows the quantification of hair follicles and the thickness of various skin layers including epidermal, cortical, muscle, and fat after treatment with MSC-CM. Among all observational groups, a significant 
increase could be seen only in the fat layer thickness, which might indicate the potential growth of the hair follicle after MSC-CM treatment (Figure 7e).

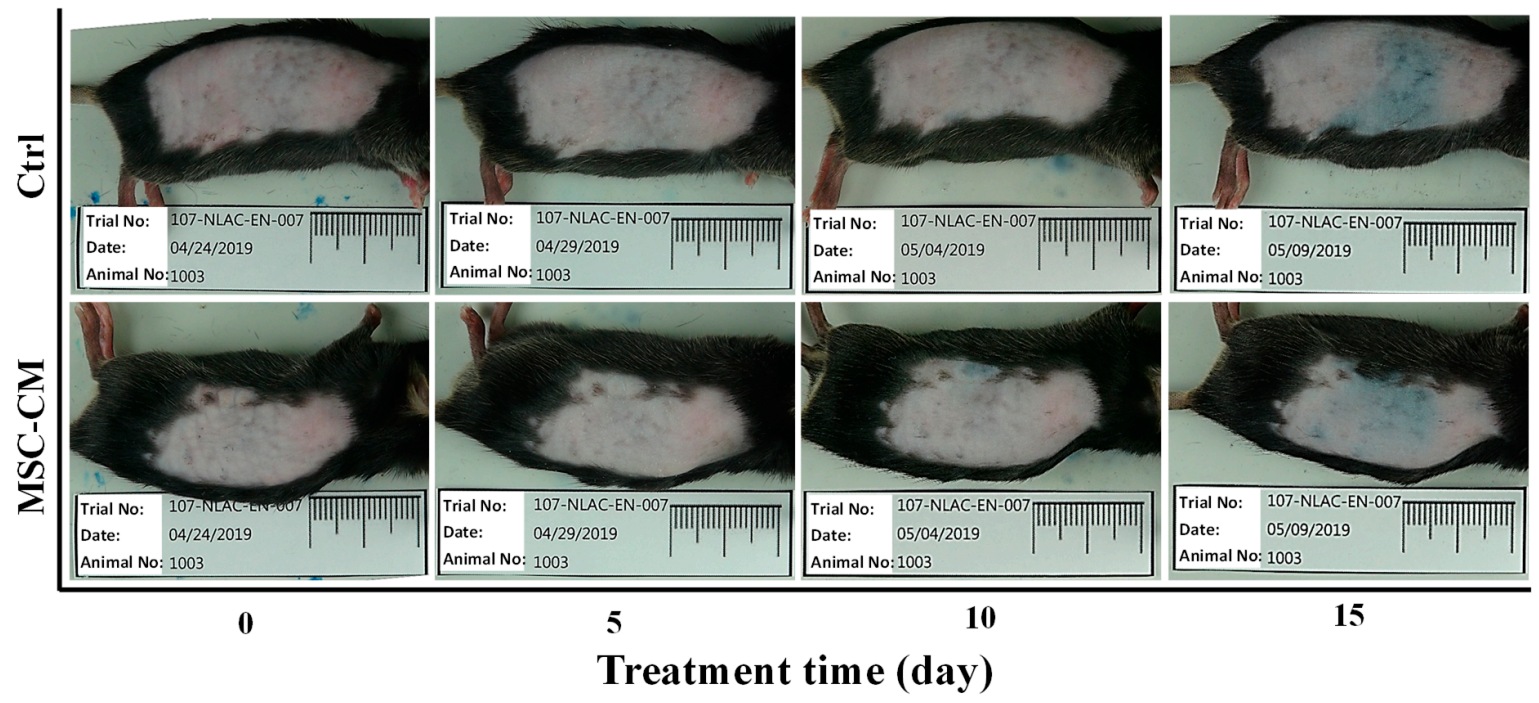

Figure 5. Animal models presenting the shaved skin of the mice after respectively being smeared with the vehicle and MSC-CM, from day 0 to day 15 of observations.

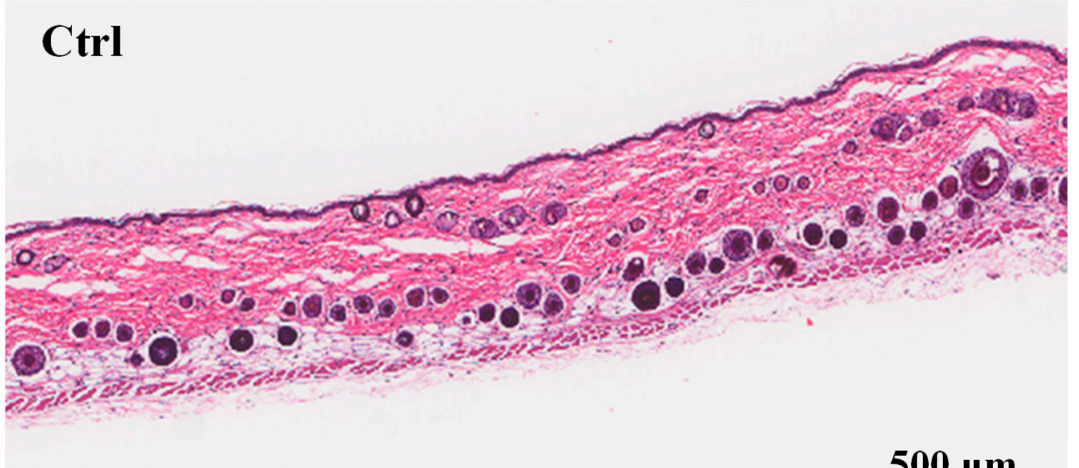

\section{MSC-CM}

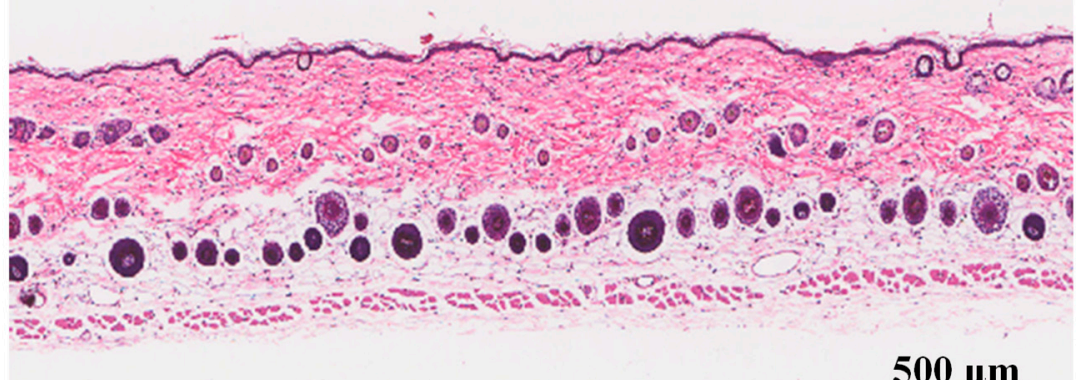

Figure 6. Histology staining of mice skins at day 15 after MSC-CM was smeared on the skin. Images from the control group (upper) and MSC-CM treated group (lower) are shown. 


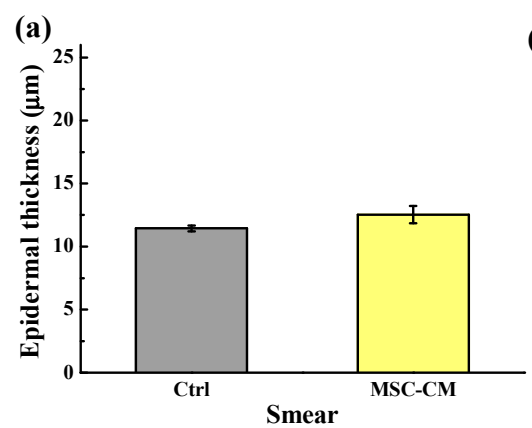

(d)

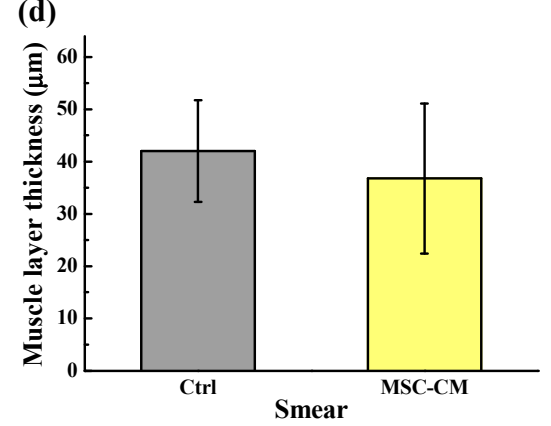

(b)

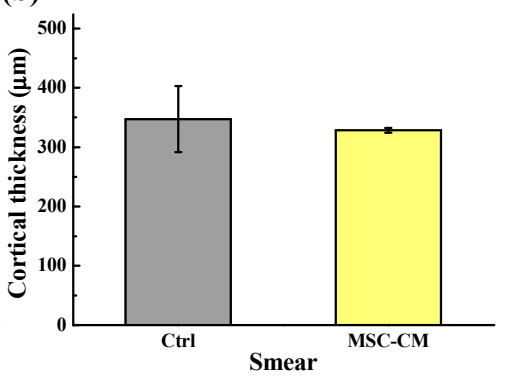

(c)

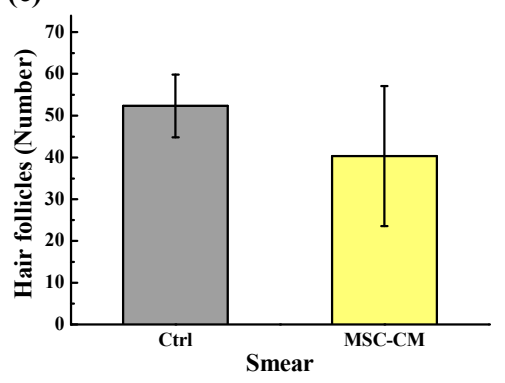

(e)

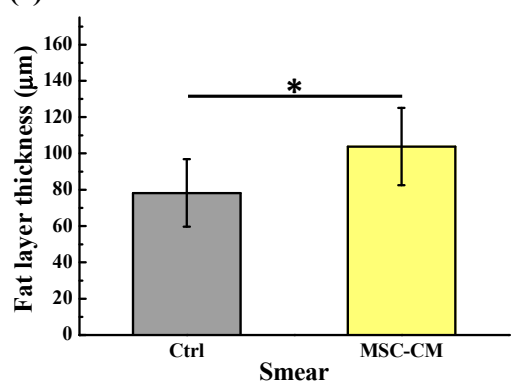

Figure 7. Quantitative evaluation showing the thickness of various skin layers after being treated with MSC-CM: (a) epidermal thickness, (b) cortical thickness, (c) hair follicles number, (d) muscle layer thickness, and (e) fat layer thickness $\left({ }^{*} p<0.05\right)$.

\section{Discussion}

In this study, we used the scratch assay to show that the migration of HaCaT keratinocytes affected wound closure in the presence of MSC-CM. It was found that wound closure was successfully achieved and covered more rapidly in almost the entire part of MSC-CM when compared to that of the control. This could be attributed to the presence of the high secretion of paracrine factors like chemokines, cytokines, and growth factors from MSC-CM that resulted in strong immunomodulatory and immunoregulatory characteristics promoting wound healing [26-28]. This feature has been indicated in the previous research that found that CM obtained from BM-MSC significantly accelerated scratch wound closure in L929 fibroblasts and HaCaT keratinocytes, supporting the application of MSC-CM as a therapeutic agent in cutaneous wound healing [26]. Accordingly, the latest study by Joseph et al. also found that MSC-CM can be used to improve the quality of wound healing with minimal scar formation [2]. Using CM from MSC culture increases fibroblast and basal cell proliferation, collagen synthesis, and improve vascularization in the process of skin wound healing $[9,11]$.

In our set of investigations, cell survival and proliferation of HaCaT keratinocyte and HFMSC during wound healing were examined in the presence of different kinds of drugs. We found that UK5099, SLL, LDHA, MSC-CM, caffeine, and CFA could promote the proliferation of HFMSC, but only MSC-CM and CFA could promote the proliferation of the HaCaT keratinocyte. Previous study found that caffeic acid produced low cytotoxicity of keratinocytes that can induce cell proliferation and differentiation [22]. Furthermore, another study confirmed that cell proliferation could also be elevated by means of CM from culture MSC [13]. In this study, we found treatment with MSC-CM exhibited the highest cell proliferation in both HFMSC and HaCaT keratinocyte during wound healing. MSC-CM could restore the proliferation and migration of keratinocytes, suggesting that MSC-CM has great potential in therapeutic modality supporting the poor wound-healing conditions and improve the effectiveness of tissue regeneration [29,30].

It has been known that wounds treated with MSCs not only displayed a sign of skin regeneration, but also showed an improvement in the formation of new skin appendages such as hair follicles and sebaceous glands [3]. The ability of skin to regenerate hair follicles during wound healing has been 
clearly demonstrated in animal models known as wound-induced hair growth (WIHG) [31-33]. In the present study, areas of the skin of the mice began to appear blue to black after respectively being smeared with MSC-CM, projecting the formation of hair follicles. For the above-mentioned reason, paracrine factors such as cytokines, chemokines, and growth factors secreted by the MSC into the medium may play a key role in activating and stimulating fibroblast proliferation during the wound healing as well as regulating human hair growth [34]. It has been known that vascular endothelial growth factor (VEGF), epidermal growth factor (EGF), fibroblast growth factor (FGF), keratinocyte growth factor (KGF), angiopoietin-1, erythropoietin, and stromal-derived factor-1 (SDF-1) escalated dermal and epidermal cell proliferation and promoted migration of dermal fibroblasts $[9,24,35]$. Furthermore, these growth factors can enhance angiogenesis and the re-epithelialization process, which might be important to stimulate hair follicle regeneration and maintain hair-inducing activity in vivo [35,36]. In addition, they are involved in the contraction phase, ending at the last stages of remodeling, leading to wound healing in the absence of scar formation and ultimately increasing hair regeneration [35]. Specifically, previous study found that Fgf9 produced from the wound promotes dermal Wnt activation and induces fibroblast Wnt expression, eventually leading to WIHG [37]. The overexpression of Wnt7 by MSC effectively promotes wound healing and at the same time, induces hair follicle regeneration through the acceleration of the biological progression of hair follicles from the telogen (resting) to anagen (growth) phase [20].

In the course of hair development, hair follicles undergo cyclic regeneration throughout its life, namely the anagen, catagen (regression), and telogen phases [38]. It has long been recognized that the adjacent tissue-layers including nerves, hair follicles, interfollicular epidermis, blood vessels, melanocytes, muscles, adipocytes, and fibroblasts participate and have a direct link in the hair cycle and formation [38,39]. Accordingly, in this study, we used the thickness of various skin layers including epidermal thickness, cortical thickness, muscle thickness, hair follicle number, and fat thickness as hair regeneration indicators. Given the limited number of samples analyzed, we could not establish a significant difference between the treated and control mice except for the fat layer thickness. Nevertheless, a previously published study found that there was a positive correlation between the thickness of the adipocyte layer with hair growth. When the hair follicles are in the anagen phase, the thickness of the adipocyte layer will rapidly increase [40]. In our study, the significant increment of the fat layer thickness found under the mouse's skin might indicate the potential growth of the hair follicle after the MSC-CM treatment. In the near future, MSC-CM may prove a promising therapeutic tool to improve wound healing and hair regeneration as well as in other regenerative applications. Specifically, the induced proliferation of stem cells present in the wound might be beneficial for the formation of new hair or prevent hair loss in alopecia patients.

\section{Conclusions}

Within the limitations of this study, we provide a new insight to necessitate the exploration of hair follicle MSC-CM as a potential therapeutic tool for wound closure as well as hair loss issues. The study confirmed that the CM from cultured HFMSC has great potential to promote cell migration and proliferation in the wound healing process, which may induce hair regeneration through changes in the wound microenvironment that affect cell-cell interactions. We believe that MSC-CM may serve as a promising alternative therapeutic modality in cell-based therapies for wound closure and alopecia. In future study, more investigations should be performed to understand the mechanism of action on which MSC-CM can stimulate wound healing in association with a positive treatment for hair loss issues.

Author Contributions: Writing-original draft, K.-L.O.; Investigation, Y.-W.K.; Data curation, C.-Y.W., F.-T.P., and Y.-C.C.; Methodology, B.-H.H.; Validation, H.-H.C.; Supervision; T.S. and T.U.; Writing-review \& editing, and M.-S.H. All authors have read and agreed to the published version of the manuscript.

Funding: The authors would like to thank the Ministry of Science and Technology, Taiwan for financially supporting this research under contract no. MOST 109-2622-8-038-005-TB1. 
Conflicts of Interest: The authors declare no conflict of interests in this work.

\section{References}

1. Dong, L.; Hao, H.; Liu, J.; Ti, D.; Tong, C.; Hou, Q.; Li, M.; Zheng, J.; Liu, G.; Fu, X.; et al. A Conditioned Medium of Umbilical Cord Mesenchymal Stem Cells Overexpressing Wnt7a Promotes Wound Repair and Regeneration of Hair Follicles in Mice. Stem Cells Int. 2017, 2017, 1-13. [CrossRef] [PubMed]

2. Joseph, A.; Baiju, I.; Bhat, I.A.; Pandey, S.; Bharti, M.; Verma, M.; Singh, A.P.; Ansari, M.M.; Chandra, V.; Saikumar, G. Mesenchymal stem cell-conditioned media: A novel alternative of stem cell therapy for quality wound healing. J. Cell. Physiol. 2020, 235, 5555-5569. [CrossRef] [PubMed]

3. De Mayo, T.; Conget, P.; Becerra-Bayona, S.M.; Sossa, C.L.; Galvis, V.; Arango, M. The role of bone marrow mesenchymal stromal cell derivatives in skin wound healing in diabetic mice. PLoS ONE 2017, 12, 0177533. [CrossRef] [PubMed]

4. Cerqueira, M.T.; Pirraco, R.P.; Marques, A.P. Stem Cells in Skin Wound Healing: Are We There Yet? Adv. Wound Care 2016, 5, 164-175. [CrossRef] [PubMed]

5. Kucharzewski, M.; Rojczyk, E.; Wilemska-Kucharzewska, K.; Wilk, R.; Hudecki, J.; Łos, M.J. Novel trends in application of stem cells in skin wound healing. Eur. J. Pharmacol. 2019, 843, 307-315. [CrossRef]

6. Hu, M.; Borrelli, M.R.; Lorenz, H.P.; Longaker, M.T.; Wan, D.C. Mesenchymal Stromal Cells and Cutaneous Wound Healing: A Comprehensive Review of the Background, Role, and Therapeutic Potential. Stem Cells Int. 2018, 2018, 6901983. [CrossRef]

7. Lee, D.E.; Ayoub, N.; Agrawal, D.K. Mesenchymal stem cells and cutaneous wound healing: Novel methods to increase cell delivery and therapeutic efficacy. Stem Cell Res. Ther. 2016, 7, 37. [CrossRef]

8. Zomer, H.D.; Varela, G.K.D.S.; Delben, P.B.; Heck, D.; Jeremias, T.D.S.; Trentin, A.G. In vitro comparative study of human mesenchymal stromal cells from dermis and adipose tissue for application in skin wound healing. J. Tissue Eng. Regen. Med. 2019, 13, 729-741. [CrossRef]

9. Payushina, O.; Butorina, N.N.; Sheveleva, O.N.; Domaratskaya, E.I. Effect of Mesenchymal Stromal Cells and Conditioned Media on Healing of Skin Wound. Bull. Exp. Boil. Med. 2018, 165, 572-575. [CrossRef]

10. Pawitan, J.A. Prospect of Stem Cell Conditioned Medium in Regenerative Medicine. BioMed Res. Int. 2014, 2014, 965849. [CrossRef]

11. Aryan, A.; Bayat, M.; Bonakdar, S.; Taheri, S.; Haghparast, N.; Bagheri, M.; Piryaei, A.; Abdollahifar, M.-A. Human Bone Marrow Mesenchymal Stem Cell Conditioned Medium Promotes Wound Healing in Deep Second-Degree Burns in Male Rats. Cells Tissues Organs 2019, 206, 317-329. [CrossRef] [PubMed]

12. Wu, Y.; Chen, L.; Scott, P.G.; Tredget, E.E. Mesenchymal Stem Cells Enhance Wound Healing Through Differentiation and Angiogenesis. STEM CELLS 2007, 25, 2648-2659. [CrossRef] [PubMed]

13. Hu, C.-H.; Tseng, Y.-W.; Chiou, C.-Y.; Lan, K.-C.; Chou, C.-H.; Tai, C.-S.; Huang, H.-D.; Hu, C.-W.; Liao, K.-H.; Chuang, S.-S.; et al. Bone marrow concentrate-induced mesenchymal stem cell conditioned medium facilitates wound healing and prevents hypertrophic scar formation in a rabbit ear model. Stem Cell Res. Ther. 2019, 10, 1-13. [CrossRef] [PubMed]

14. Kober, J.; Gugerell, A.; Schmid, M.; Zeyda, M.; Buchberger, E.; Nickl, S.; Hacker, S.; Ankersmit, H.J.; Keck, M. Wound Healing Effect of Conditioned Media Obtained From Adipose Tissue on Human Skin Cells. Ann. Plast. Surg. 2016, 77, 156-163. [CrossRef]

15. Sun, B.; Guo, S.; Xu, F.; Wang, B.; Liu, X.; Zhang, Y.; Xu, Y. Concentrated Hypoxia-Preconditioned Adipose Mesenchymal Stem Cell-Conditioned Medium Improves Wounds Healing in Full-Thickness Skin Defect Model. Int. Sch. Res. Not. 2014, 2014, 1-6. [CrossRef]

16. Hsu, L.-C.; Peng, B.-Y.; Chen, M.-S.; Thalib, B.; Ruslin, M.; Tung, T.D.X.; Chou, H.-H.; Ou, K.-L. The potential of the stem cells composite hydrogel wound dressings for promoting wound healing and skin regeneration: In vitro and in vivo evaluation. J. Biomed. Mater. Res. Part B Appl. Biomater. 2018, 107, 278-285. [CrossRef]

17. Sun, J.; Zhang, Y.; Song, X.; Zhu, J.; Zhu, Q. The Healing Effects of Conditioned Medium Derived from Mesenchymal Stem Cells on Radiation-Induced Skin Wounds in Rats. Cell Transplant. 2018, 28, $105-115$. [CrossRef]

18. Flores, A.; Schell, J.; Krall, A.S.; Jelinek, D.; Miranda, M.; Grigorian, M.; Braas, D.; White, A.C.; Zhou, J.L.; Graham, N.A.; et al. Lactate dehydrogenase activity drives hair follicle stem cell activation. Nat. Cell Biol. 2017, 19, 1017-1026. [CrossRef] 
19. Martinez-Outschoorn, U.; Prisco, M.; Ertel, A.; Tsirigos, A.; Lin, Z.; Pavlides, S.; Wang, C.; Flomenberg, N.; Knudsen, E.S.; Howell, A.; et al. Ketones and lactate increase cancer cell "stemness", driving recurrence, metastasis and poor clinical outcome in breast cancer: Achieving personalized medicine via Metabolo-Genomics. Cell Cycle 2011, 10, 1271-1286. [CrossRef]

20. Dong, L.; Hao, H.; Xia, L.; Liu, J.; Ti, D.; Tong, C.; Hou, Q.; Han, Q.; Zhao, Y.; Liu, H.; et al. Treatment of MSCs with Wnt1a-conditioned medium activates DP cells and promotes hair follicle regrowth. Sci. Rep. 2014, 4, 5432. [CrossRef]

21. Shushtari, N.; Froushani, S.M.A. Caffeine Augments The Instruction of Anti-Inflammatory Macrophages by The Conditioned Medium of Mesenchymal Stem Cells. Cell J. 2017, 19, 415. [PubMed]

22. Kim, B.; Kim, J.E.; Kim, H.-S. Caffeic acid induces keratinocyte differentiation by activation of PPAR- $\alpha$. J. Pharm. Pharmacol. 2013, 66, 84-92. [CrossRef] [PubMed]

23. Wang, X.; Tredget, E.E.; Wu, Y. Dynamic Signals for Hair Follicle Development and Regeneration. Stem Cells Dev. 2012, 21, 7-18. [CrossRef] [PubMed]

24. Chen, L.; Tredget, E.E.; Wu, P.Y.G.; Wu, Y. Paracrine Factors of Mesenchymal Stem Cells Recruit Macrophages and Endothelial Lineage Cells and Enhance Wound Healing. PLoS ONE 2008, 3, e1886. [CrossRef] [PubMed]

25. Yoon, B.S.; Moon, J.-H.; Jun, E.K.; Kim, J.; Maeng, I.; Kim, J.S.; Lee, J.H.; Baik, C.S.; Kim, A.; Cho, K.S.; et al. Secretory Profiles and Wound Healing Effects of Human Amniotic Fluid-Derived Mesenchymal Stem Cells. Stem Cells Dev. 2010, 19, 887-902. [CrossRef]

26. Walter, M.; Wright, K.; Fuller, H.R.; MacNeil, S.; Johnson, W. Mesenchymal stem cell-conditioned medium accelerates skin wound healing: An in vitro study of fibroblast and keratinocyte scratch assays. Exp. Cell Res. 2010, 316, 1271-1281. [CrossRef]

27. Im, G.-B.; Kim, Y.H.; Kim, Y.-J.; Kim, S.-W.; Jung, E.; Jeong, G.-J.; Wang, K.; Kim, J.; Kim, D.-I.; Kim, T.-H.; et al. Enhancing the Wound Healing Effect of Conditioned Medium Collected from Mesenchymal Stem Cells with High Passage Number Using Bioreducible Nanoparticles. Int. J. Mol. Sci. 2019, 20, 4835. [CrossRef]

28. Mehanna, R.; Nabil, I.; Attia, N.; Bary, A.A.; Razek, K.A.; Ahmed, T.A.E.; Elsayed, F. The Effect of Bone Marrow-Derived Mesenchymal Stem Cells and Their Conditioned Media Topically Delivered in Fibrin Glue on Chronic Wound Healing in Rats. BioMed Res. Int. 2015, 2015, 1-12. [CrossRef]

29. Li, M.; Luan, F.-X.; Zhao, Y.; Hao, H.; Liu, J.; Dong, L.; Fu, X.; Han, W. Mesenchymal stem cell-conditioned medium accelerates wound healing with fewer scars. Int. Wound J. 2015, 14, 64-73. [CrossRef]

30. Jun, E.K.; Zhang, Q.; Yoon, B.S.; Moon, J.-H.; Lee, G.; Park, G.; Kang, P.J.; Lee, J.H.; Kim, A.; You, S. Hypoxic Conditioned Medium from Human Amniotic Fluid-Derived Mesenchymal Stem Cells Accelerates Skin Wound Healing through TGF- $\beta / S M A D 2$ and PI3K/Akt Pathways. Int. J. Mol. Sci. 2014, 15, $605-628$. [CrossRef]

31. Rahmani, W.; Liu, Y.; Rosin, N.L.; Kline, A.; Raharjo, E.; Yoon, J.; Stratton, J.A.; Sinha, S.; Biernaskie, J. Macrophages Promote Wound-Induced Hair Follicle Regeneration in a CX3CR1- and TGF- $\beta 1-D e p e n d e n t$ Manner. J. Investig. Dermatol. 2018, 138, 2111-2122. [CrossRef] [PubMed]

32. Yuriguchi, M.; Aoki, H.; Taguchi, N.; Kunisada, T. Pigmentation of regenerated hairs after wounding. J. Dermatol. Sci. 2016, 84, 80-87. [CrossRef] [PubMed]

33. Wier, E.M.; Garza, L.A. Through the lens of hair follicle neogenesis, a new focus on mechanisms of skin regeneration after wounding. Semin Cell Dev Biol. 2020, 100, 122-129. [CrossRef] [PubMed]

34. Gunawardena, T.N.A.; Masoudian, Z.; Rahman, M.T.; Ramasamy, T.S.; Ramanathan, A.; Abu Kasim, N.H. Dental derived stem cell conditioned media for hair growth stimulation. PLoS ONE 2019, 14, e0216003. [CrossRef] [PubMed]

35. Alexandrescu, V. Wound Healing: New Insights into Ancient Challenges; BoD-Books on Demand: London, UK, 2016.

36. Rendl, M.; Polak, L.; Fuchs, E. BMP signaling in dermal papilla cells is required for their hair follicle-inductive properties. Genome Res. 2008, 22, 543-557. [CrossRef]

37. Gay, D.; Kwon, O.S.; Zhang, Z.; Spata, M.; Plikus, M.V.; Holler, P.D.; Ito, M.; Yang, Z.; Treffeisen, E.; Kim, C.-D.; et al. Fgf9 from dermal $\gamma \delta \mathrm{T}$ cells induces hair follicle neogenesis after wounding. Nat. Med. 2013, 19, 916-923. [CrossRef]

38. Balañá, M.E.; Charreau, H.E.; Leirós, G.J. Epidermal stem cells and skin tissue engineering in hair follicle regeneration. World J. Stem Cells 2015, 7, 711-727. [CrossRef] 
39. Chase, H.B.; Montagna, W.; Malone, J.D. Changes in the skin in relation to the hair growth cycle. Anat. Rec. Adv. Integr. Anat. Evol. Boil. 1953, 116, 75-81. [CrossRef]

40. Donati, G.; Proserpio, V.; Lichtenberger, B.M.; Natsuga, K.; Sinclair, R.; Fujiwara, H.; Watt, F.M. Epidermal Wnt/ $\beta$-catenin signaling regulates adipocyte differentiation via secretion of adipogenic factors. Proc. Natl. Acad. Sci. USA 2014, 111, E1501-E1509. [CrossRef]

(C) 2020 by the authors. Licensee MDPI, Basel, Switzerland. This article is an open access article distributed under the terms and conditions of the Creative Commons Attribution (CC BY) license (http://creativecommons.org/licenses/by/4.0/). 